\title{
Thermo-mechanical characterization of epoxy nanocomposites with different carbon nanotube distributions obtained by solvent aided and direct mixing
}

\author{
A. Martone, C. Formicola, F. Piscitelli, M. Lavorgna, M. Zarrelli", V. Antonucci, M. Giordano \\ CNR - Research National Council, IMCB - Institute for Composite and Biomedical Materials, P.le E. Fermi 1, 80055 \\ Portici, Italy
}

Received 24 October 2011; accepted in revised form 19 January 2012

\begin{abstract}
Two different routes, namely solvent aided dispersion and direct mixing, were employed to disperse MultiWalled Carbon Nanotubes (MWNTs) into a mono-component epoxy system used as matrix for advanced composites. In the first route, MWCNTs were diluted in three different solvents (acetone, sodium dodecyl sulfate and ethanol) and then mixed with the matrix by tip sonication. In the second case, carbonaceous nanoparticles were added directly into the hosting system and dispersion was carried out by using three different techniques (mechanical stirring, magnetic agitation and tip sonication). The effects of the solvents and agitation energy were investigated by optical microscopy at micron level, in order assess the more efficient dispersion procedure for the considered epoxy system. It was demonstrated that parameters associated with direct mixing rather than solvent solubility govern MWCNT dispersion. Optical analysis of the nanocomposite morphology evidenced a very low density of MWCNTs micron sized aggregates in the case of direct mixed tip sonicated samples if compared to those obtained by solution aided dispersion. In addition, nanocomposites obtained by sonication showed the lowest density of MWCNTs micron sized aggregates, also when compared with mechanically and magnetically stirred system.

Dynamic Mechanical Analysis (DMA) and Thermo-Mechanical Analysis (TMA) results confirm the final result that among the considered direct mixing techniques, the direct tip sonication represents the most efficient route for MWCNT dispersion. Moreover, the mixing temperature of the hosting matrix system represents a fundamental feature in enhancing the MWCNT de-bundling and dispersion.

Small X-ray Scattering analysis revealed that a nanosized structure of nanotubes is formed in the case of the tip sonicated samples that is heuristically correlated with both the maximum enhancement of mechanical modulus and the maximum reduction of thermal expansion coefficients.
\end{abstract}

Keywords: nanocomposites, processing technologies, thermosetting resins

\section{Introduction}

The continuous demand for new high performance polymer composite for sectors as aerospace or lightweight structures for various structural elements has lead many researchers to investigate the potential application of the CNT as nano-reinforcements for polymer matrix of fiber-reinforced composite. Polymer/CNT composites have attracted consider- able attention due to their unique mechanical, surface, multi-functional properties and strong interactions with the matrix, depending on the nano-scale microstructure and large interfacial area [1-5]. Moreover, their impressive mechanical properties with stiffness and strength values falling within the range 100-1800 GPa and 2.5-3.5 GPa respectively, make them ideal candidates to develop novel com-

\footnotetext{
*Corresponding author, e-mail: mauro.zarrelli@imcb.cnr.it

(c) BME-PT
} 
posites based on advanced polymer matrices [6-7]. Epoxy nanocomposite matrix could be the ideal solution for a new generation of composite materials: at mesoscopic level, for example, to attenuate the mismatch of thermal expansion coefficient between the reinforcement and the matrix system or to enhance matrix-based property; while at macrolevel to reduce the development of residual stresses due different volume variations between the tooling materials and composite element. The dispersion process of carbon nanotubes represents a very complex phenomenon due to their natural tendency to bundle together due to van der Waals interactions. Nanotube attraction and, thus, bundle formation, are based on chemical and physical features: for single CNT, in fact, the attractive energy is of the order of $0.5 \mathrm{eV}$ (about $0.8 \cdot 10^{-19} \mathrm{~J}$ ) per nanometer of nanotube-to-nanotube contact [8] at ambient temperature, for a $10 \mathrm{~nm}$ nanotube length this force increases to the order of $200 \mathrm{~kJ} / \mathrm{mol}$, which is equivalent to a typical covalent bond. The non-uniform dispersion due to microscopic nanotubes aggregates leads to many defect sites and resin- rich area limiting not only the efficiency of CNTs as reinforcement fillers for the polymer composite matrix [912], but also the electrical properties and the percolation threshold [13-15]. In the published literature [16-20], various techniques have been reported to disperse nanotubes in either thermosets or thermoplastics polymer resins. Among others, solvent aided dispersion and direct mixing stand as the most capable and efficient methodologies. Solution dispersion can be considered a suitable technique [21, 22], especially in the case of thermosets, even though some difficulties could arise from the choice of the most appropriate solvent and processing conditions (i.e. temperature and time). Solvent aided dispersion is carried out generally by a two-stage procedure, which includes firstly, the dispersion of nanotubes in the selected solvent (solvent mixing) and, then, the addition of the resin for the final mixing (resin solution mixing). Among different solvents, acetone and ethanol are the most common materials enabling the carbon nanotubes dispersion. The effect of acetone and ethanol on the properties of SWCNTs $(0.5 \mathrm{wt} \%)$ epoxy nanocomposites was investigated in [22]. In particular, it was found that the acetone-SWCNTs dispersed samples are characterized by greater size of SWNT bundles diameter and, conversely, higher values of hardness and flexural strength that those of ethanol-SWCNTs dispersed samples. Further, to disaggregate and uniform disperse carbon nanotubes, another extensive studied approach is the use of surfactants [23] by altering the surface chemistry of the tubes either covalently (functionalization) or non-covalently (adsorption). Ionic surfactants are preferable for CNT/ water-soluble solutions. Alternatively, non-ionic surfactants are proposed when organic solvents have to be used. Among the ionic surfactants, sodium dodecyl sulphate (SDS) and dodecyl-benzene sodium-sulfonate (NaDDBS) were commonly used to decrease CNT aggregative tendency in water. In particular, SDS is characterized by an hydrophilic and hydrophobic head at its end. Diluted in a water solution, SDS operates a separation of the nanotube agglomerates by intercalating the carbonaceous nano-structures [23] and the water molecules by chemical interaction at its ends. This effect will be lost during the degassing procedure as dense nanotube aggregations will form, while the solvent evaporates and the resin lowers its viscosity. Direct mixing operates directly on the mixture of (pristine or purified) nanotubes and hosting system, without any pre-dispersion stage. This single-stage procedure leads to a nanotubes/resin mixture directly used as matrix for composite materials. Various mixing techniques (i.e. magnetic stirring, roll milling, mechanical agitation or high-energy sonication) have been employed for both procedures to achieve dispersion and homogenization of CNT within the polymer matrix. However, achieved results in terms of repetitive morphology and performance of the final nanocomposite are still controversial and scattered $[24,25]$. Nanomechanics and characterization testing of nanocomposite sample have shown that enhancement of the mechanical and electrical behaviour of the final nanocomposite is strongly dependent upon the level of dispersion and the final morphology of the nanofillers.

A review of the available literature data on mechanical elastic modulus of nanofilled resin was reported in [24]. Successful modelling of the data variability was achieved by introducing two fundamental features associated to nanofiller dispersed within a hosting matrix such as their effective aspect ratio (i.e. actual length over nominal diameter of the nanostructures) and excluded volume. Figure 1 


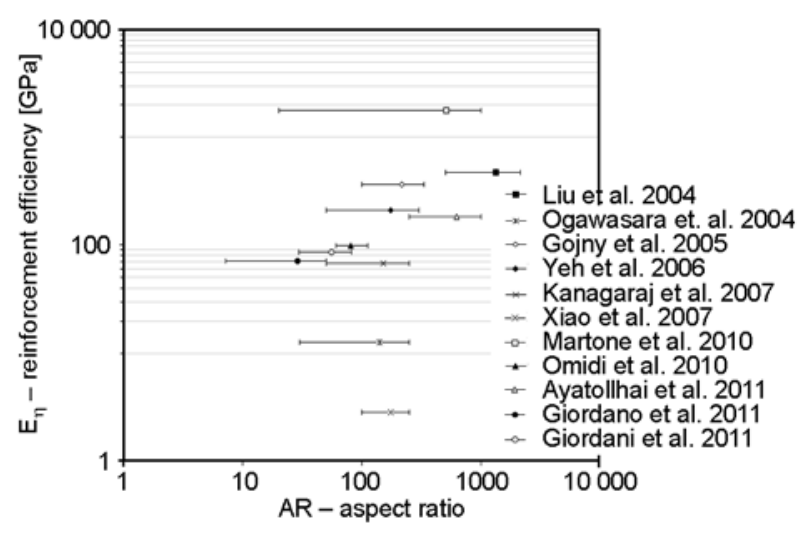

Figure 1. Available reinforcement efficiency data from 2004 to 2011 [25]

reports the reinforcement efficiencies of different experimental data found in the literature from 2004 to 2010. In the case of a thermally activated resin, the possible activation of the resin cure is a further issue, which may compromise the efficiency of the dispersion procedure, thus it needs to be taken into account. The final stage of mixing, either for solution dispersion or direct mixing, indeed, represents a critical phase due to the local raising of the temperature which may lead to the potential triggering of the cure reaction. An accurate control of the temperature and a suitable characterization of the rheological behaviour of the hosting matrix allow the identification of the most suitable temperature window preserving the unreacted state of the thermosetting matrix.

The aim of the present paper is to evaluate the effect of different mixing procedures on the resulting morphology of carbon nanotubes micrometric aggregates and, in turn, on the thermo-mechanical properties of the final epoxy matrix nanocomposite. The main novelty of this work is related to the explanation of the major efficiency of one technique (direct tip sonication) compared to the other ones, mainly investigating two principal features: potential application of this technique for scaled up process and achieved reinforcement efficiency of the nanofillers in the final nanocomposite. The adopted nanotube content corresponds to the statistical percolation threshold $(0.1 \% \mathrm{wt} / \mathrm{wt})$ according to previously published data for the same resin and nanotube type [25]. Statistical percolation threshold represent the minimum content at which the nanofiller give arise to a percolative network within the hosting matrix thus effecting the overall macroscopic property of the final materials, i.e. percolation, ad example, represents the switching limit for these kind of system between electrical isolation and conductive behaviour. The dispersion of untreated MWCNTs into the chosen monocomponent epoxy has been performed by either using different solvents ( $\mathrm{sol}$ vent aided dispersion) and also by means of different agitation energy mechanisms (direct mixing). Preliminary thermal analysis and rheological tests were performed on the neat resin system in order to optimize the processing conditions in terms of the viscosity of the hosting system and 'dwell' period. The dispersion states of CNTs within the nanocomposite samples obtained by different dispersion routes have been characterised on the micron scale by optical microscopy $(\mathrm{OM})$ and on the sub micrometric scale by Small Angle X-ray Scattering technique. Optical microscopy analysis of the nanocomposite morphology evidenced a very low density of MWCNTs micron sized aggregates in the case of direct mixed tip sonicated samples compared to nanocomposites obtained by solution aided dispersion technique and low energy mixing (magnetic and mechanical agitation). Small Angle X-ray Scattering analysis revealed that only in the case of tip sonicated samples carbon nanotubes are organised on a nanometric scale net. Such morphological feature corresponds on the macroscale to the highest increase of the bending elastic modulus and to the highest reduction of the thermal expansion coefficients among the manufactured nanocomposites.

\section{Materials}

The 7000 grade MWNTs carbon nanotubes, provided by Nanocyl S.A. (Belgium), were adopted. These nanotubes are grown by catalytic chemical vapordeposition (CCVD) with a purity of $90 \%$, a mean length of 10 micron and a mean diameter of $10 \mathrm{~nm}$. These MWNTs were used directly without any purification. A commercial premixed epoxy-amine resin, denoted as RTM6, was used as matrix. This system, already degassed, fulfils the requirements of the aerospace industries in advanced liquid injection moulding processes and it was provided by Hexcel Composites (Duxford, UK). The solvent used in this work (acetone, ethanol and the sodium dodecyl sulphate) were acquired by Sigma Aldrich. 


\section{Preparation of MWCNT/epoxy samples}

A fixed percentage content of MWCNT $(0.1 \% \mathrm{w} / \mathrm{w})$ has been used for the preparation of nanocomposites. The chosen percentage corresponds to the statistical percolation threshold considering the nominal aspect ratio of the nanotube $(L / d \sim 1000)$ as reported in [25]. In the case of solution dispersion, nanoparticles were dispersed in about $40 \mathrm{~mL}$ of solvent in a sealed beaker by an ultrasonic process (power has been set at $18 \mathrm{~W}$ ), cooled with ice, for 30 minutes at room temperature. Then, the epoxy resin ( $40 \mathrm{gr})$ was added to the nanotubes solution, immersed in an oil bath at $80^{\circ} \mathrm{C}$ and sonicated for further 30 minutes until the removal of solvent residual content. The final solution was, then, poured in an aluminium mould, vacuum degassed for $30^{\prime}$ at $90^{\circ} \mathrm{C}$ and then cured. For all nanocomposite samples, the cure schedule was 1.5 hour at $160^{\circ} \mathrm{C}$ and 2 hours at $180^{\circ} \mathrm{C}$ to assure the same conditions for comparison purpose.

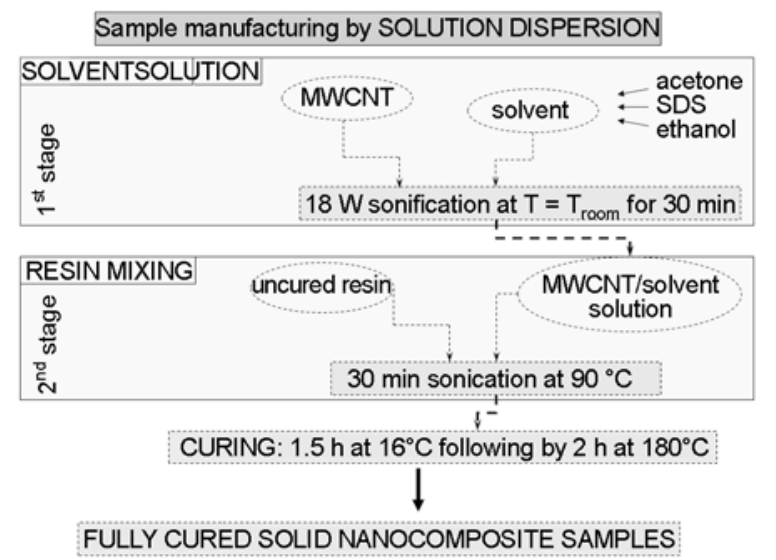

Figure 2. Flow chart of sample manufacturing by solution dispersion
The procedure, presented as direct mixing, was characterised by the following stages: pristine nanotube were added directly to the hosting epoxy matrix (40 gr) to perform the mixing stage at constant temperature $\left(60,90\right.$ and $\left.120^{\circ} \mathrm{C}\right)$ for 60 minutes. In this case, the preliminary dispersion using the solvent was not carried out, but analogous conditions for the resin mixing, degassing and final polymerization were set. Magnetic agitation was performed by using FALC F60 (Falc Instruments, Italy) system equipped with a $20 \mathrm{~W}$ motor power and standard bar (15 mm lenght at $150 \mathrm{rpm}$ ); the mechanical stirring was performed by using a Heidolph RZR 2020 stirrer $(50 \mathrm{~W}$ powered motor by Heidolph Instruments GmbH \& Co. KG, Germany) equipped with a viscojet head and set at $1050 \mathrm{rpm}$. A Misonix S3000 sonicator (Misonix Inc., New York, USA) equipped with a titanium tapped horn with a 1/2" $(12.7 \mathrm{~mm})$ diameter tip was employed for the sonication stage. The process flow charts for each dispersion route are presented schematically in Figures 2 and 3.

\section{Experimental methods}

Optical microscopy analysis was carried out by using an Olympus system type BX51 (Olympus Italia S.r.l., Milan, Italy) in transmitted light configuration, to operate a preliminary classification of the nanotube dispersion. Differential Scanning Calorimetry (DSC) analysis has been performed by TA DSC instrument type 2920 (TA Instruments. Milan, Italy) with temperature ranging from -50 to $310^{\circ} \mathrm{C}$ at different heating rates and isothermal temperatures. The viscosity of the neat resin system was measured during cure, in both isothermal and

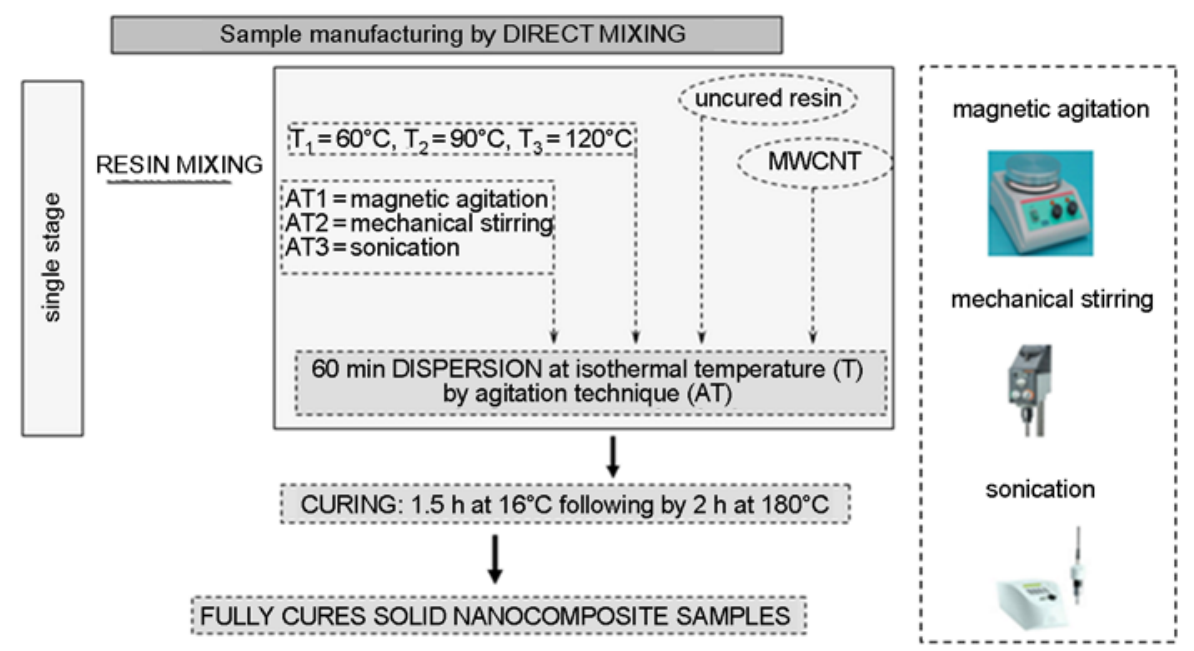

Figure 3. Flow chart of sample manufacturing by direct mixing at three different temperatures $\left(T_{1}, T_{2}\right.$ and $\left.T_{3}\right)$ by three agitation techniques (AT1, AT2, and AT3) 
dynamic conditions by using an AntonPaar Instruments Rheometer (Anton Paar GmbH, Germany). The resin was drop onto a $40 \mathrm{~mm}$ parallel plate system with the bottom plate and the top plate (gap $=$ $0.5 \mathrm{~mm}$ ) oscillating at a fixed frequency of $0.5 \mathrm{~Hz}$. Thermo-mechanical tests were performed by using a thermo-mechanical analyzer type TM60-WS from Shimadzu (M. Penati Strumenti srl, Milan, Italy), equipped with a macro-tip. The TMA scans were carried out on samples with rectangular cross section $(3 \mathrm{~mm} \times 3 \mathrm{~mm})$ and maximum length of $15 \mathrm{~mm}$ according to the ISO 113598 , with a $2^{\circ} \mathrm{C} / \mathrm{min}$ temperature ramp. For each scan, the sample was placed in the cell and temperature equilibrated at $30^{\circ} \mathrm{C}$ for 10 minutes; linear dimension was monitored as the temperature arises up to $280^{\circ} \mathrm{C}$. Nanocomposite specimens with nominal dimension of $60 \times 10 \times 2 \mathrm{~mm}$ were mechanically tested under three points bending mode by a TA 2980 Dynamic Mechanical Analyzer (TA Instruments, Milan, Italy), within the linear elastic region at given sub-glassy transition temperature $\left(80^{\circ} \mathrm{C}\right)$ with an amplitude varying within the range from 20 to $60 \mu \mathrm{m}$. Wide and Small Angle X-ray Scattering (WAXS and SAXS) were performed with a SAXSess Instrument (Anton Paar $\mathrm{GmbH}$, Germany), equipped with a 2D imaging plate detection system by using copper $K_{\alpha}$ radiation $\lambda=0.1542 \mathrm{~nm}$. The X-Ray generator was operated at $40 \mathrm{kV}$ and $50 \mathrm{~mA}$ and a line collimation was used to ensure high radiation intensity. All scattering data, dark current, background and incoherent scattering were subtracted, normalized for the primary beam intensity and finally desmeared. The scattering profile related to that of the neat epoxy resin was used as background for the composite scattering profiles.

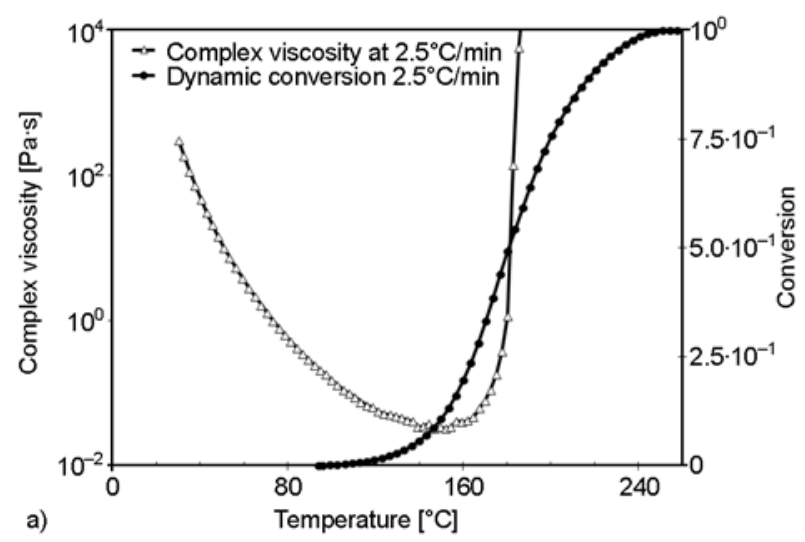

\section{Results and discussion}

\subsection{Chemo-rheological characterization of neat epoxy}

Mono-component thermosetting polymers undergo a polymerization reaction, triggered by the temperature, which transforms the material from a liquid monomeric system to a fully reacted solid-like structure. During the curing, the viscosity of the system abruptly changes, reaching, theoretically, infinite values whenever the system crosses to the gelation point. Analysis of polymerization kinetics, therefore, is the most fundamental experimental data to be acquired before any mixing procedure could be attempted and validated.

A preliminary characterization of the neat epoxy system was performed to evaluate the chemo-rheological behaviour. The chemorheology at $5^{\circ} \mathrm{C} / \mathrm{min}$ has been reported in Figure 4, as shows the minimum viscosity occurs around $120^{\circ} \mathrm{C}$, which corresponds to the limiting processing temperature. Isothermal DMA characterization at $120^{\circ} \mathrm{C}$, reported in Figure 4b, confirms that the system is very slowly reacting. According to previously reported data on the same epoxy system [25], a 'dwell' time of $60 \mathrm{~min}$ to mix the resin would not significantly affect the system viscosity.

\subsection{Optical analysis of nanocomposites}

Optical microscopy images have been qualitatively analyzed in terms of two main parameters: particles size and homogeneity of the sample, i.e. nanotubes clusters size and their spatial distribution through the sample. As a reference Figure 5 shows the micrographs of the nanocomposite samples obtained by hand mixing processed for $30 \mathrm{~min}$ at constant temperatures $\left(60\right.$ and $\left.90^{\circ} \mathrm{C}\right)$.

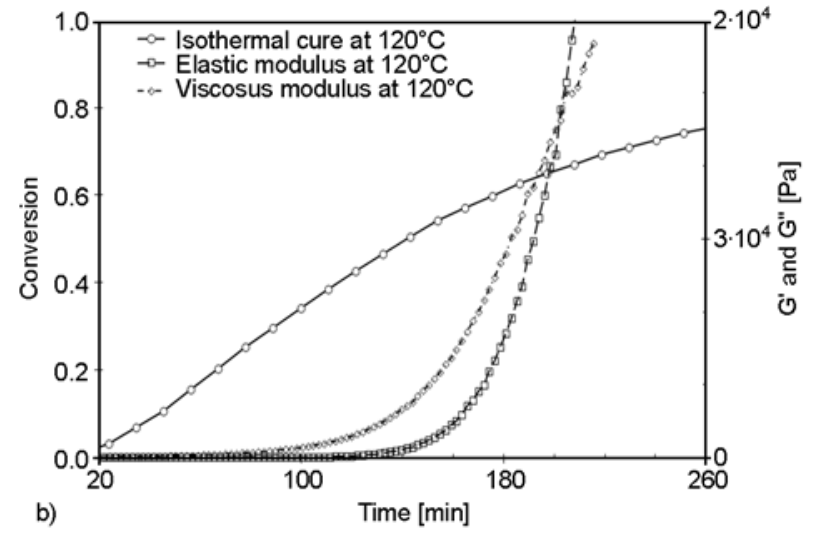

Figure 4. RTM6 chemorheology at $5^{\circ} \mathrm{C} / \mathrm{min}$ a) and at $120^{\circ} \mathrm{C}$ isothermal temperature b) 


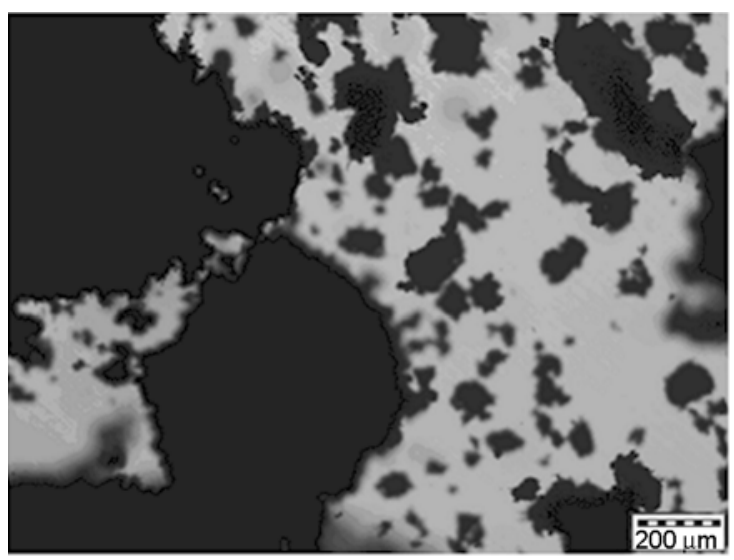

a)

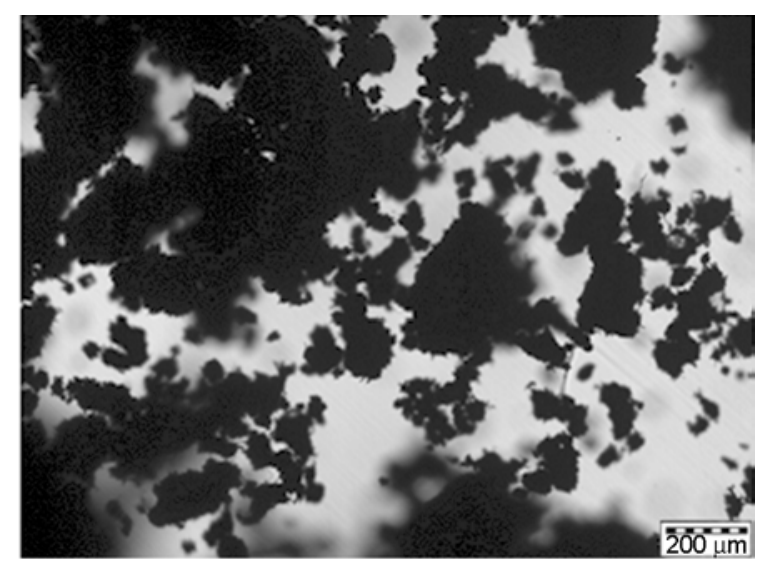

b)

Figure 5. Optical image of nanocomposite obtained by hand-dispersion at $60^{\circ} \mathrm{C}$ a) and $90^{\circ} \mathrm{C} \mathrm{b}$ )

The nanotube agglomerates are preserved in their pristine shape appearing as unswollen. Hand mixing procedure led to a very poor level of pristine aggregates dispersion and homogenization with dense dark nanotube clusters surrounded by bright rich resin areas at both temperatures.

\subsubsection{Solution dispersed samples}

Different solvents were considered: acetone, ethanol and sodium dodecyl sulfate surfactant in water to improve the unwrapping and de-bundling the nanotubes agglomerates before adding them into the resin system.

The resin mixing with nanotubes/solvent solution was carried out by heating the oil bath up to $80^{\circ} \mathrm{C}$ and then holding this temperature for 30 minutes: higher temperatures would drastically reduce the available time for mixing before the solvents evaporation, while lower temperatures would induce a higher viscosity of the hosting system.

Table 1 reports details of all performed solution dispersion procedures. Optical micrographs are presented in Figure 6a, 6b and 6c for nanocomposite samples processed by solution dispersion and in Figure $6 \mathrm{~d}$ for by sonication direct mixing at $60^{\circ} \mathrm{C}$. Dispersion in acetone (Figure 6a) led to large CNT agglomerates characterised by indistinct bounds with a denser central core. The average dimension of the agglomerations is of the order of $100 \mu \mathrm{m}$ of the darker area. Optical images of samples dispersed in water/SDS (Figure 6b) show some residuals of solvent within a matrix of tiny spherical-shaped microagglomerates with an average diameter of few micrometers. In this case, the dispersion efficiency is quite promising although remarks remain either for the possibility to degas out completely the solvent content and for the chemical nature of the solvent during the dispersion solution. In the case of ethanol (Figure 6c), a coarse morphology is visible that is characterised by micro-sized nanotube agglomerates. Large semi-transparent areas can be noticed highlighting the sub optical resolution of dispersed nanotubes. A micrograph of a nanocomposite specimen obtained by tip sonication, without solvent, at $60^{\circ} \mathrm{C}$ is reported in Figure 6d. Very few nanotube aggregates are visible with sizes of few microns, very smaller than the pristine aggregates dimensions. The major part of the nanocomposite is semi-transparent and homogeneous, thus indicating that nanotubes are dispersed within the matrix forming CNT agglomerates, undetectable by the optical microscopy. In conclusion, optical microscopy demonstrate that by employing the tip sonication technique, without solvent, allows to obtain nano-

Table 1. Solution mixing procedure by using three different solvents

\begin{tabular}{|l|c|c|c|c|}
\hline & Solvent/MWCNT solution & Resin mixing & Degassing & Curing \\
\hline Figure 6a & $\begin{array}{c}30^{\prime} \text { at } T_{\text {room }} \text { ultrasonication } \\
\text { at } 24 \mathrm{~W} \text { in acetone }\end{array}$ & $\begin{array}{c}30^{\prime} \text { at } 80^{\circ} \mathrm{C}, \\
\text { ultrasonication at } 24 \mathrm{~W}\end{array}$ & 60 min at $90^{\circ} \mathrm{C}$ under vacuum & $1.5 \mathrm{~h}$ at $160^{\circ} \mathrm{C} 2 \mathrm{~h}$ at $180^{\circ} \mathrm{C}$ \\
\hline Figure 6b & $\begin{array}{c}30^{\prime} \text { at } T_{\text {room ultrasonication }} \text { at } 24 \mathrm{~W} \text { in water/SDS } \\
\text { ultrasonication at } 24 \mathrm{~W}\end{array}$ & 60 min at $90^{\circ} \mathrm{C}$ under vacuum & $1.5 \mathrm{~h}$ at $160^{\circ} \mathrm{C} 2 \mathrm{~h}$ at $180^{\circ} \mathrm{C}$ \\
\hline Figure $6 \mathrm{c}$ & $\begin{array}{c}30^{\prime} \text { at } T_{\text {room }} \text { ultrasonication } \\
\text { at } 24 \mathrm{~W} \text { in ethanol }\end{array}$ & $\begin{array}{c}30^{\prime} \text { at } 80^{\circ} \mathrm{C} \\
\text { ultrasonication at } 24 \mathrm{~W}\end{array}$ & 60 min at $90^{\circ} \mathrm{C}$ under vacuum & $1.5 \mathrm{~h}$ at $160^{\circ} \mathrm{C} 2 \mathrm{~h}$ at $180^{\circ} \mathrm{C}$ \\
\hline
\end{tabular}




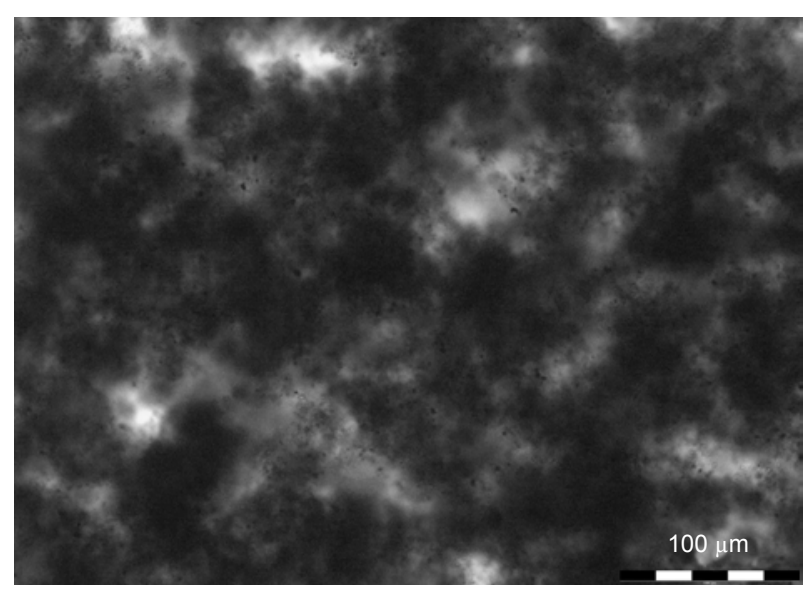

a)

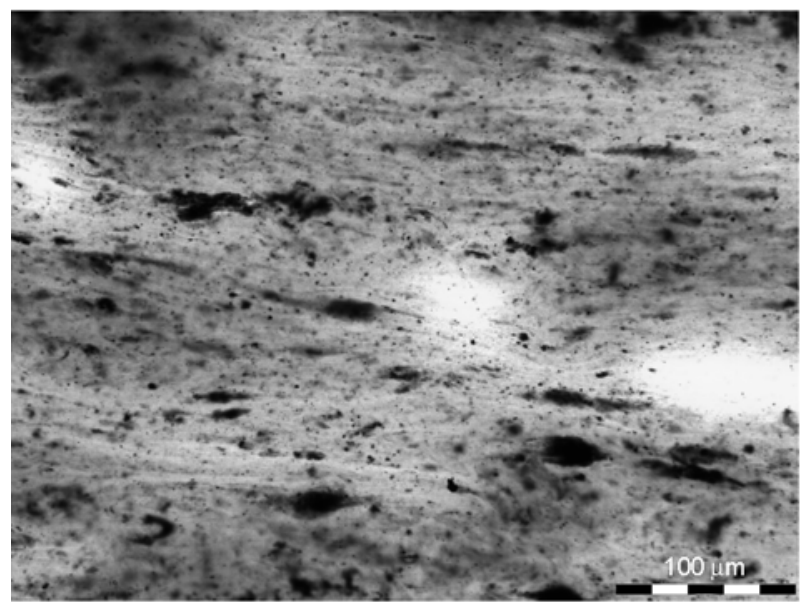

c)

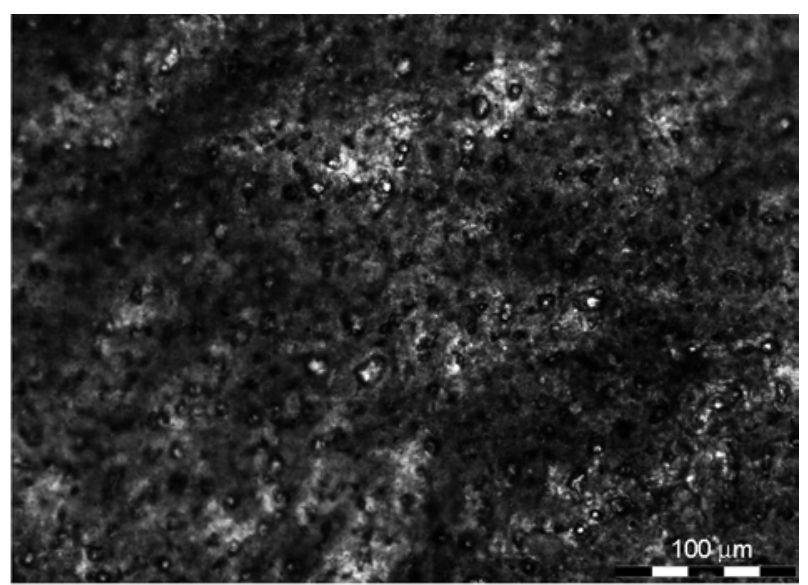

b)

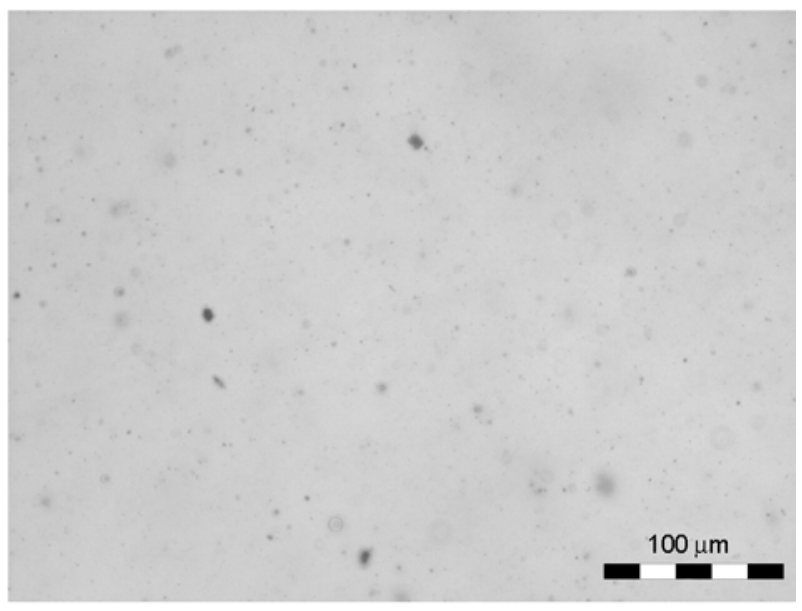

d)

Figure 6. Optical microscopy for solution dispersed samples in (a) acetone (b) surfactant (c) ethanol and (d) direct mixed nanocomposite

composites with remarkable uniformity in CNT distribution and a sub-micron level of nanostructure texture.

\subsubsection{Direct mixed samples}

The direct mixing of MWCNT in RTM6 was performed at three different temperatures corresponding to three different levels of viscosity of the hosting system, 66, 190 and $692 \mathrm{mPa} \cdot \mathrm{s}$ respectively 120,90 and $60^{\circ} \mathrm{C}[25]$.

The containing beaker was immersed in an oil bath and opportunely temperature controlled until mixing temperature level was reached. Figure 7 reports a 'mosaic' of optical images for each used techniques. The disposition of the images in a 3 by 3 matrix shows the final composite morphology evolution with temperatures $\left(60,90\right.$ and $\left.120^{\circ} \mathrm{C}\right)$ and with the employed mixing techniques (magnetic, mechanical and sonication). At all three considered temperatures, magnetic and mechanical stirring lead optically highly non-homogeneous samples where nanotube agglomerates have dimensions lower than that of the hand mixed reference sample. In particular, mechanically mixed nanocomposites show lower sized micrometric nanotube clusters and a more homogeneous distribution of such clusters than the magnetically mixed samples. On the contrary nanocomposites obtained by sonication mixing are optically homogeneous with rare micron sized aggregates whose density decreases with the temperature. Nanotubes clusters are rarely optically visible at minimum viscosity temperature $\left(120^{\circ} \mathrm{C}\right)$. For the first two mixing techniques, the temperature level does not much affect the final dispersion state.

\subsection{X-ray analysis of nanocomposites obtained by direct mixing}

The SAXS and WAXS profiles of epoxy matrix and both nanocomposites prepared by sonication, mechanical stirring and magnetical agitation at 


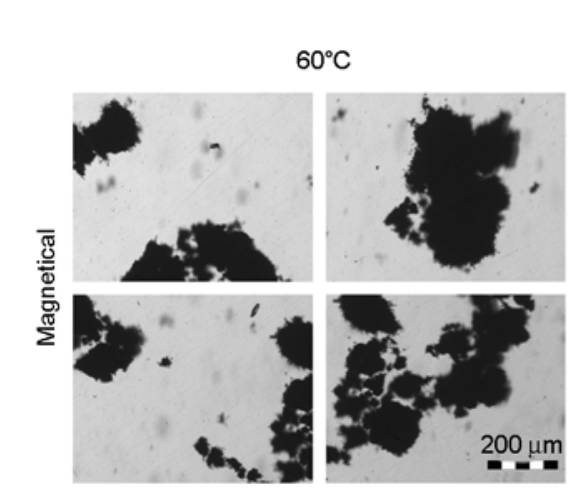

a)

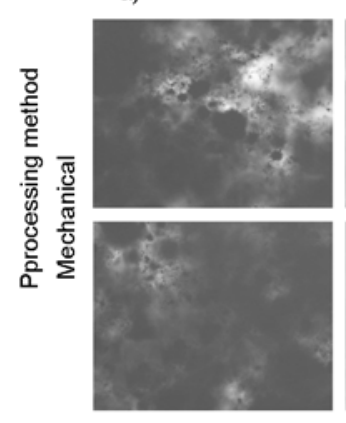

d)

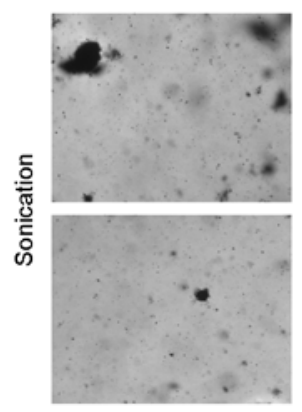

g)
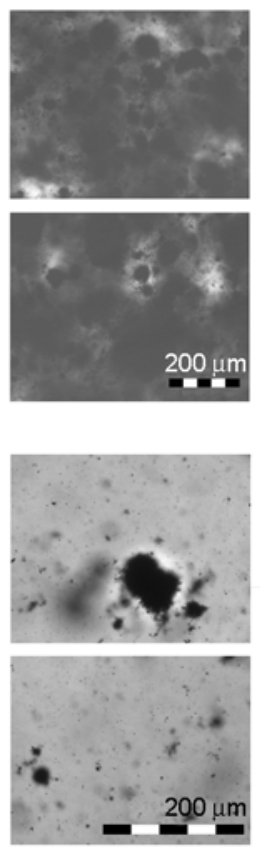

(n)

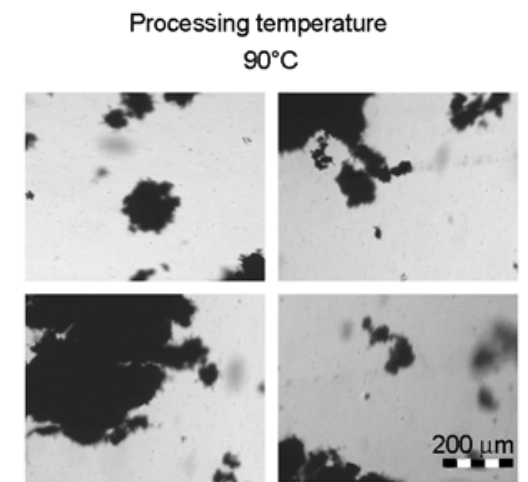

b)

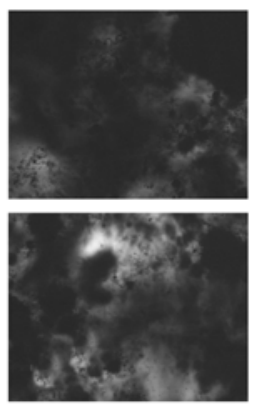

e)

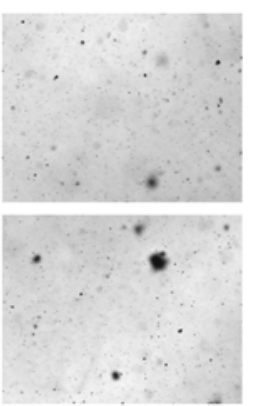

h)
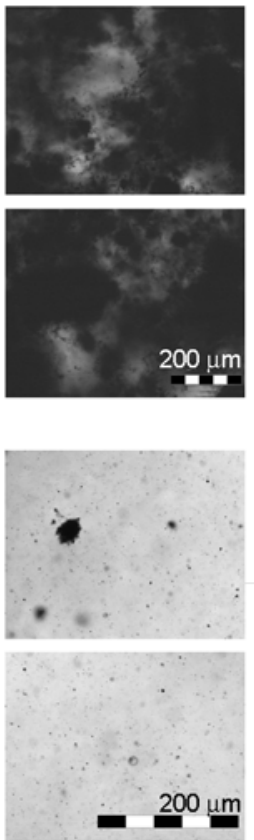

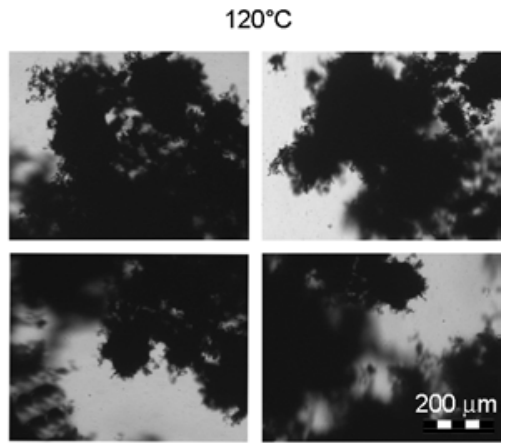

c)

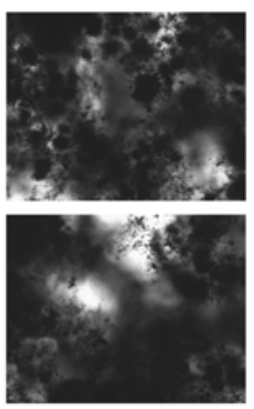

f)

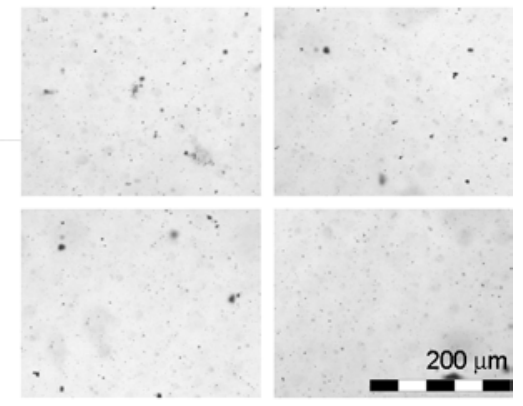

i)

Figure 7. Optical images of nanocomposites prepared at various $T$ by different mixing techniques
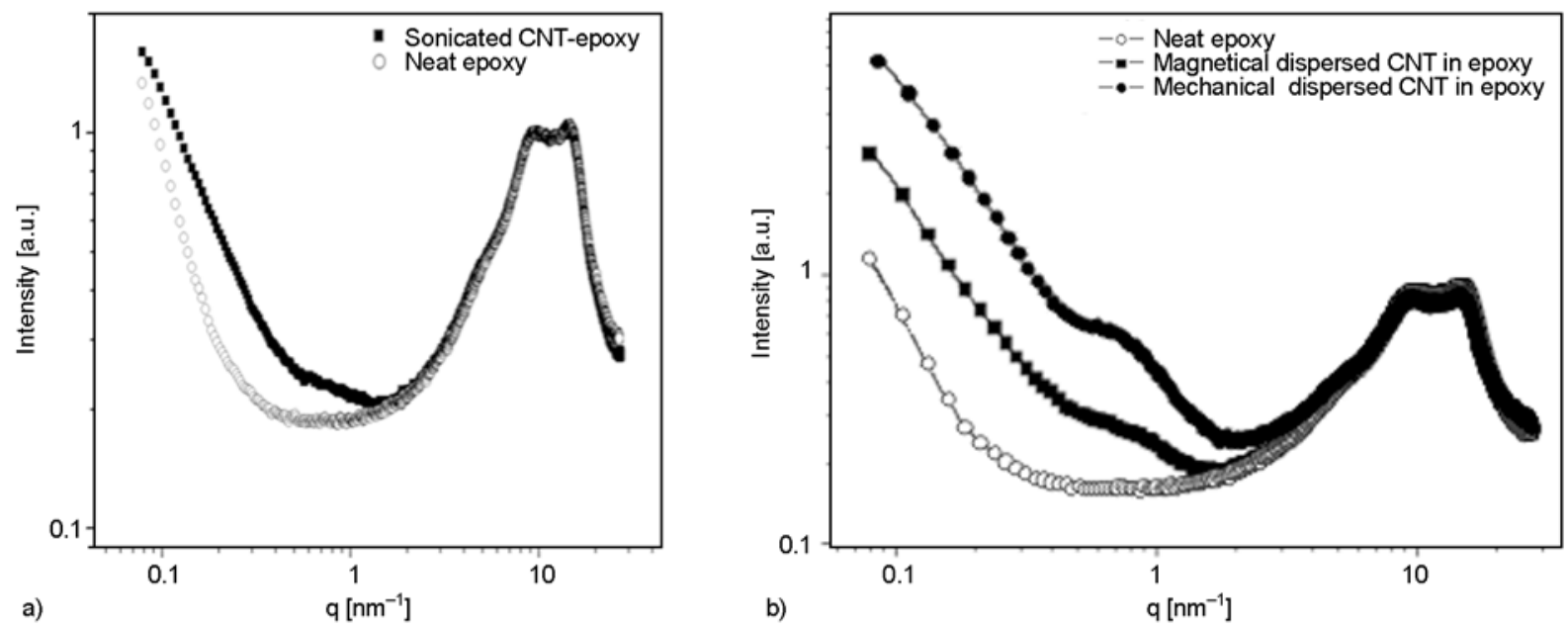

Figure 8. Scattering profiles for nanocomposites obtained by sonication vs neat system (a) and magnetical agitation, mechanical stirring vs neat system (b) 


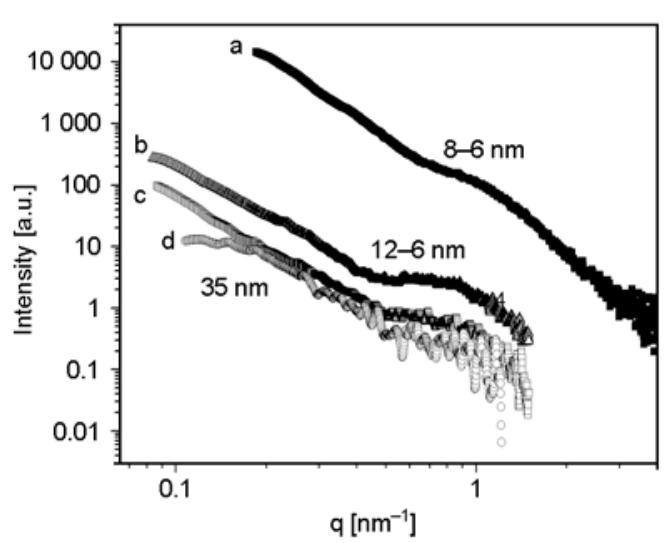

Figure 9. SAXS intensity Porod plots of the background a) and sample obtained by magnetic (b), mechanical (c) and sonication (d) dispersion methods

$120^{\circ} \mathrm{C}$ are shown in Figure 8. It is worth noting that regardless the preparation procedure the nanocomposites display the typical halo featuring with the neat epoxy resin centred around $10 \mathrm{~nm}^{-1}$.

Since the presence of CNTs does not affect the intimate structure of epoxy matrix, the neat epoxy scattering profile can be used as background subtracted to the composites profiles, thus isolating the CNT contribution. The scattering profile related to the neat epoxy resin was superimposed. In the inset an enlargement at low q values is reported. The SAXS profiles of CNTs within composites prepared at $120^{\circ} \mathrm{C}$ by using the three above mentioned direct mixing methods are shown in Figure 9, as log-log Porod plot of the scattering intensity, $I$ versus $q$.

The plot of CNT powders used to prepare the composites is also reported for comparison in Figure 9. The scattering profile of CNT powders is shown as comparison (a). The CNTs powders profile showed a knee-scattering feature corresponding to a size of

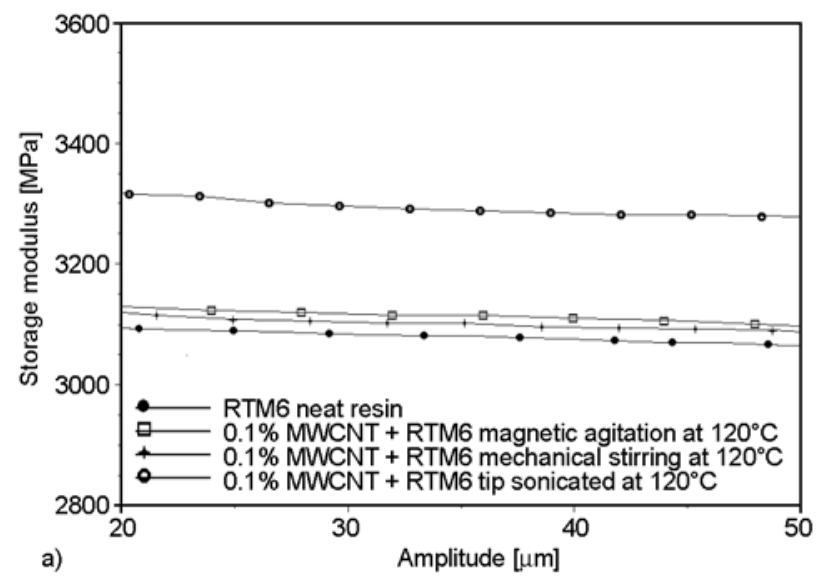

Figure 10. DMA tests performed on nanocomposite samples processed at $120^{\circ} \mathrm{C}$ by using three mixing techniques a) and flexural moduli for nanocomposite samples processed by direct mixing at $120^{\circ} \mathrm{C} \mathrm{b}$ ) about $6-8 \mathrm{~nm}$, assessed as $d=2 \pi / q[26,27]$. Since the mean diameter of CNT was about $10 \mathrm{~nm}$, it could be reasonably thought that this dimension corresponds to the cross-section of CNT when they were analysed vs. air, i.e. in powder state. The same characteristic feature is also highlighted in the scattering profiles of composites prepared by mechanical and magnetic dispersion mode, confirming the poor dispersion of CNTs bundles in the epoxy matrix as appeared by microscope analyses. On the other hand, in the scattering profile of the sonicated composite, it is worth noting a strong reduction of above mentioned knee-feature, together with an additional knee-scattering feature at about $0.18 \mathrm{~nm}^{-1}$, corresponding to a dimension of about $35 \mathrm{~nm}$. As previously reported [24] this size could be associated to a bundle consisting of about 3 nanotubes side-by-side interacting.

\subsection{Mechanical and dilatometric analysis of nanocomposites obtained by direct mixing}

Dynamic mechanical tests were performed on samples manufactured by direct mixing at $120^{\circ} \mathrm{C}$ to evaluate the bending modulus. Figure 10 a reports the strain sweep scans of the three nanocomposite samples processed at the same isothermal temperature $\left(120^{\circ} \mathrm{C}\right)$ by means of the different energy shearbased techniques. Figure $10 \mathrm{~b}$ reports the flexural modulus and $40 \mu \mathrm{m}$ amplitude. Flexural modulus of the neat system was found equal to $2998 \pm 96.5 \mathrm{MPa}$, according to previously reported data, whilst sonicated samples revealed the maximum modulus enhancement of about $10 \%(3285 \pm 338.1 \mathrm{MPa})$. Flexural modulus of nanocomposite samples obtained by magnetic or mechanical mixing results slightly

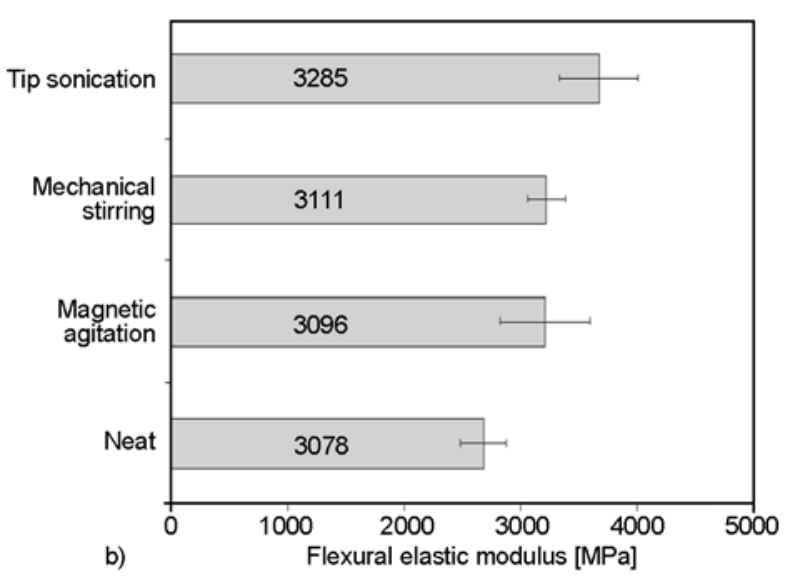


higher compared to the neat resin and respectively, equal to $3096 \pm 384.6$ and $3111 \pm 164.2 \mathrm{MPa}$.

Morphology of the reinforcing phase, i.e. nanotubes clusters size and spatial distribution through the sample, strongly affects the flexural modulus of the nanocomposite. In particular, the presence of micron sized clusters of nanotubes has a detrimental effect of the flexural modulus. Reduction of clusters size leads to an improvement of the nanocomposite modulus.

Sonication will act the in direction of disaggregate the nanotube bundles giving arise to a submicrolevel texture due to the cavitation effect induced by ultrasound waves. Moreover, the cooperative motion due to the density gradient induced by the cavitation under the sonicator tip leads to a uniform spatial distribution of the nanofiller within the hosting system. For these reasons, it seems reasonable to assume that a better impregnation of the carbon nanotube will amplify the effect of stress transfer under loaded condition by the overall final nancomposite. On the other hand, large microsized clusters formed during the mechanical or magnetic agitation can present a different content of resin thus lowering the stress transfer efficiency with the matrix. It can be argued that microsized formation and therefore contractedness between of nanofiller will inevitably reduce the reinforcement efficiency. In fact, while for electrical behaviour contacts between tubes improve electron transport, their occurrence will inevitably reduce the nominal length of the nanofiller (CNT length is further reduced by the sonication effect, as reported in the literature) thus strongly affecting the stress transfer with the matrix. The positive effect associated with sonication technique could be reviewed mainly in

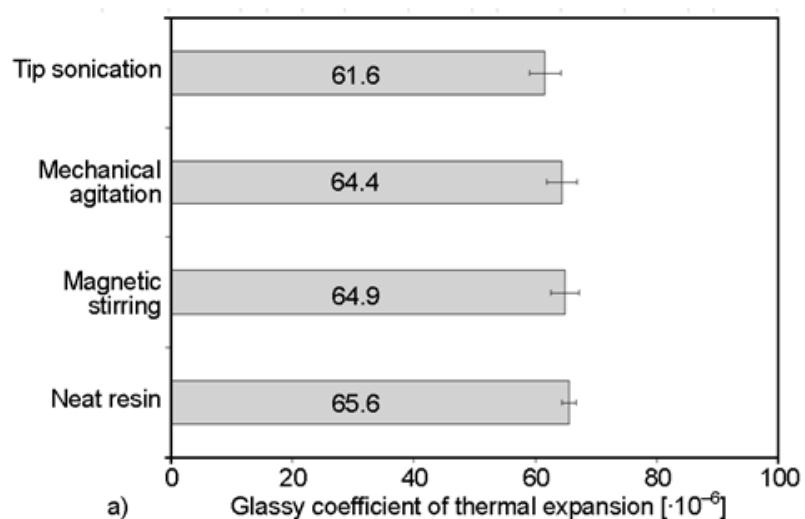

two fundamental features: firstly, sonication and high shear induced by the cavitation strongly disaggregate the microsized structure of pristine nanotubes thus enhancing the available area for stress transfer, whereas the CNT length reduction will act in the opposite direction. Concurrently also sample homogeneity (i.e. the spatial distribution of the nanotube filler within the matrix) plays a role in determining the nanocomposite mechanical modulus. Neat epoxy and nanocomposite samples were also tested by thermo-mechanical analyser (TMA) to evaluate the coefficient of thermal expansions (CTE), in both rubber and glassy regions [27]. Figure 11 shows the linear expansion dilatometric curves for the nanocomposite samples processed by direct mixing at isothermal temperature of $120^{\circ} \mathrm{C}$ along with the corresponding curve of neat epoxy system.

In the glassy region, all nanocomposites presented lower thermal dilatations than neat epoxy. Maximum reduction of about $6 \%$ has been found in the case of sonication produced samples. More accentu-

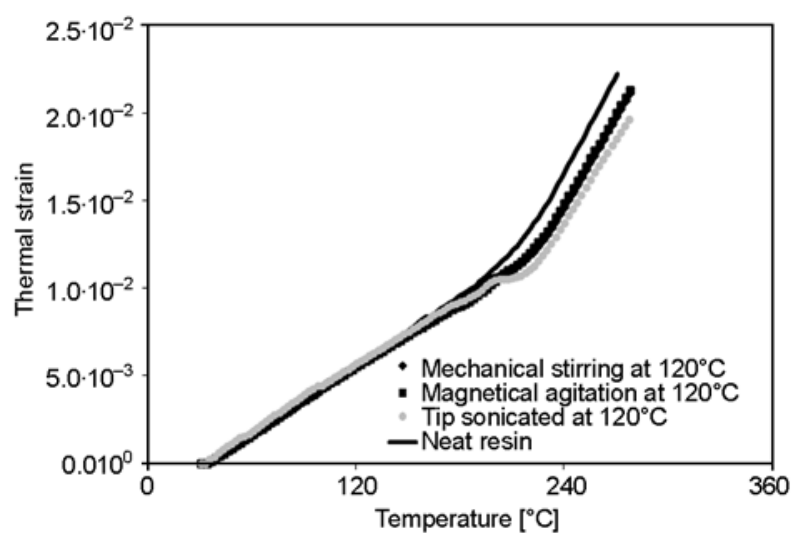

Figure 11. TMA scans of direct mixed nanocomposite samples under and above glass transition temperature

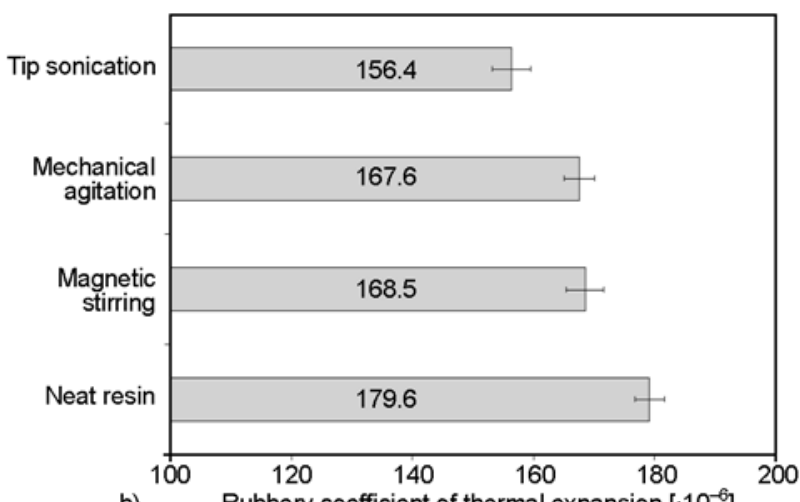

Figure 12. Bar diagram of coefficient of thermal expansion for the nanocomposite samples processed by direct mixing procedure a) rubbery and b) glassy values 
ated effects have been observed in the rubbery coefficient of thermal expansion.

The values, in fact, result much lower for the nanocomposite processed by tip-sonication with an average variation of around $13 \%$; while a change of about $5 \%$ is measured for the nanocomposite processed by magnetic and mechanical technique (Figure 12a and $b$ ).

\section{Conclusions}

Different dispersion procedures have been considered to disperse multi-walled carbon nanotubes within a commercial epoxy resin used as matrix for advanced composites namely solvent aided dispersion and direct mixing. The effect of selected solvents and mixing techniques on the final nanocomposite morphology has been qualitatively investigated with reference to the presence of micron sized nanotubes aggregates by optical microscopy. It was found that preliminary stage using solvent aided dispersion can be suitably avoided, simplifying the scaling-up of the dispersion procedure at industrial level. The final nanocomposite morphology by direct sonication leads to a submicron and more uniform texture compared to the others. This was mainly attributed to the different mechanisms involved into the dispersion process by using high-energy ultrasonication: a) tip cavitation which induces pristine nanotube bundles disgregation and $b$ ) the overall convective motions of the resin due to the density gradient under the sonication tip which homogenises the spatial distribution of the dispersed nanofiller within the hosting matrix.

Small Angle X-ray diffraction analysis revealed that the sonication aided mixing produces nanocomposites with nanometric sized structures (bundles of $35 \mathrm{~nm}$ of diameter at $120^{\circ} \mathrm{C}$ ) and a very low density of carbon nanotubes aggregates. As expected, the bending modulus of the nanocomposite increases with the reduction of the density of micrometric carbon nanotubes aggregates due to the higher reinforcement efficiency achieved in the final nanocomposite. In addition, the rubbery thermal expansion coefficient of the sonication produced sample showed also a significant reduction of $15 \%$ in respect to the matrix value where samples produced by lower energy manufacturing routes (magnetic and mechanical stirring) showed a modest reduction of $5 \%$.

\section{References}

[1] Baughman R. H., Zakhidov A. A., de Heer W. A.: Carbon nanotubes - The route toward applications. Science, 297, 787-792 (2002).

DOI: $10.1126 /$ science. 1060928

[2] Coleman J. N., Khan U., Blau W. J., Gun'ko Y. K.: Small but strong: A review of the mechanical properties of carbon nanotube-polymer composites. Carbon, 44, 1624-1652 (2006).

DOI: 10.1016/j.carbon.2006.02.038

[3] Xie X. L., Mai Y. W., Zhou X. P.: Dispersion and alignment of carbon nanotubes in polymer matrix: A review. Materials Science and Engineering: R: Reports, 49, 89-112 (2005).

DOI: $10.1016 /$ j.mser.2005.04.002

[4] Ajayan P. M., Schadler L. S., Giannaris C., Rubio A.: Single-walled carbon nanotube-polymer composites: Strength and weakness. Advanced Materials, 12, 750753 (2000).

DOI: 10.1002/(SICI)1521-4095(200005)12:10<750:: AID-ADMA750>3.3.CO;2-Y

[5] Schadler L. S., Giannaris S. C., Ajayan P. M.: Load transfer in carbon nanotube epoxy composites. Applied Physics Letters, 73, 3842-3844 (1993).

DOI: $10.1063 / 1.122911$

[6] Tibbetts G. G., Beetz C. P.: Mechanical properties of vapour-grown carbon fiber. Journal of Physics D: Applied Physics, 20, 292-297 (1987).

DOI: $10.1088 / 0022-3727 / 20 / 3 / 008$

[7] Martone A., Formicola C., Giordano M., Zarrelli M.: Reinforcement efficiency of multi-walled carbon nanotube/epoxy nano composites. Composites Science and Technology, 70, 1154-1160 (2010).

DOI: $10.1016 /$ j.compscitech.2010.03.001

[8] Dyke C. A., Tour J. M.: Covalent functionalization of single-walled carbon nanotubes for materials applications. Journal of Physical Chemistry A, 108, 1115111159 (2004).

DOI: $10.1021 / \mathrm{jp} 046274 \mathrm{~g}$

[9] Qian D., Dickey E. C., Andrews R., Rantell T.: Load transfer and deformation mechanisms in carbon nanotube-polystyrene composites. Applied Physics Letters, 76, 2868-2871 (2000).

DOI: $10.1063 / 1.126500$

[10] Park C., Ounaies Z., Watson K. A., Crooks R. E., Smith J., Lowther S. E., Connell J. W., Siochi E. J., Harrison J. S., St. Clair T. L.: Dispersion of single wall carbon nanotubes by in situ polymerization under sonication. Chemical Physics Letters, 364, 303-308 (2002). DOI: 10.1016/S0009-2614(02)01326-X

[11] Ham H. T., Choi Y. S., Chung I. J.: An explanation of dispersion states of single-walled carbon nanotubes in solvents and aqueous surfactant solutions using solubility parameters. Journal of Colloid and Interface Science, 286, 216-223 (2005).

DOI: $10.1016 /$ j.jcis.2005.01.002 
[12] Salvetat J-P., Briggs G. A. D., Bonard J-M., Bacsa R. R., Kulik A. J., Stöckli T., Burnham N. A., Forró L.: Elastic and shear moduli of single-walled carbon nanotube ropes. Physical Review Letters, 82, 944-947 (1999).

DOI: 10.1103/PhysRevLett.82.944

[13] Choi E. S., Brooks J. S., Eaton D. L., Al-Haik M. S., Hussaini M. Y., Garmestani H., Li D., Dahmen K.: Enhancement of thermal and electrical properties of carbon nanotube polymer composites by magnetic field processing. Journal of Applied Physics, 94, 6034 6039 (2003).

DOI: $10.1063 / 1.1616638$

[14] Sandler J., Shaffer M. S. P., Prasse T., Bauhofer W., Schulte K., Windle A. H.: Development of a dispersion process for carbon nanotubes in an epoxy matrix and the resulting electrical properties. Polymer, 40, 59675971 (1999).

DOI: 10.1016/S0032-3861(99)00166-4

[15] Sandler J. K. W., Kirk J. E., Kinloch I. A., Shaffer M. S. P., Windle A. H.: Ultra-low electrical percolation threshold in carbon-nanotube-epoxy composites. Polymer, 44, 5893-5899 (2003).

DOI: 10.1016/S0032-3861(03)00539-1

[16] Lau K-T., Lu M., Lam C-K., Cheung H-Y., Sheng FL., Li H-L.: Thermal and mechanical properties of single-walled carbon nanotube bundle-reinforced epoxy nanocomposites: The role of solvent for nanotube dispersion. Composites Science and Technology, 65, 719 725 (2005).

DOI: 10.1016/j.compscitech.2004.10.005

[17] Li J., Tong L., Fang Z., Gu A., Xu Z.: Thermal degradation behavior of multi-walled carbon nanotubes/ polyamide 6 composites. Polymer Degradation and Stability, 91, 2046-2052 (2006).

DOI: 10.1016/j.polymdegradstab.2006.02.001

[18] Wang Z., Liang Z., Wang B., Zhang C., Kramer L.: Processing and property investigation of single-walled carbon nanotube (SWNT) buckypaper/epoxy resin matrix nanocomposites. Composites Part A: Applied Science and Manufacturing, 35, 1225-1232 (2004). DOI: $10.1016 /$ j.compositesa.2003.09.029

[19] Wu J., Chung D. D. L.: Calorimetric study of the effect of carbon fillers on the curing of epoxy. Carbon, 42, 3039-3042 (2004).

DOI: $\underline{10.1016 / \mathrm{j} . \text { carbon.2004.07.010 }}$
[20] Xu X., Thwe M. M., Shearwood C., Liao K.: Mechanical properties and interfacial characteristics of carbon-nanotube-reinforced epoxy thin films. Applied Physics Letters, 81, 2833-2835 (2002).

DOI: $10.1063 / 1.1511532$

[21] Lau K-T., Lu M., Lam C-K., Cheung H-Y., Sheng FL., Li H-L.: Thermal and mechanical properties of single-walled carbon nanotube bundle-reinforced epoxy nanocomposites: The role of solvent for nanotube dispersion. Composites Science and Technology, 65, 719-725 (2005). DOI: 10.1016/j.compscitech.2004.10.005

[22] Vaisman L., Wagner H. D., Marom G.: The role of surfactants in dispersion of carbon nanotubes. Advances in Colloid and Interface Science, 128-130, 37-46 (2005).

DOI: $10.1016 /$ j.cis.2006.11.007

[23] Faiella G., Piscitelli F., Lavorgna M., Antonucci V., Giordano M.: Tuning the insulator to conductor transition in a multi walled carbon nanotubes/epoxy matrix composite at sub statistical percolation threshold. Applied Physics Letters, 95, 153106/1-153106/3 (2009). DOI: $10.1063 / 1.3242017$

[24] Martone A., Faiella G., Antonucci V., Giordano M., Zarrelli M.: Investigation of the effective reinforcement modulus of carbon nanotubes in an epoxy matrix. in 'Carbon nanotubes - From research to applications' (ed.: Bianco S.) InTech Publisher, Rijeka, 269294 (2011).

[25] Martone A., Faiella G., Antonucci V., Giordano M., Zarrelli M.: The effect of the aspect ratio of carbon nanotubes on their effective reinforcement modulus in an epoxy matrix. Composites Science and Technology, 71, 1117-1123 (2011). DOI: 10.1016/j.compscitech.2011.04.002

[26] Hernández J. J., García-Gutiérrez M. C., Nogales A., Rueda D. R., Ezquerra T. A.: Small-angle X-ray scattering of single-wall carbon nanotubes dispersed in molten poly(ethylene terephthalate). Composites Science and Technology, 66, 2629-2632 (2006). DOI: 10.1016/j.compscitech.2006.05.008

[27] Pizzutto C. E., Suave J., Bertholdi J., Pezzin S. H., Coelho L. A. F., Amico S. C.: Mechanical and dilatometric properties of carboxylated SWCNT/epoxy composites: Effects of the dispersion in the resin and in the hardener. Journal of Reinforced Plastics and Composites, 29, 524-530 (2010).

DOI: $10.1177 / 0731684408099924$ 